\title{
Study of Hadron Scattering Using an Asymmetric Box
}

\author{
C. Liu*; Y. Shen, X. Li, G.Z. Meng, X. Feng, M. Gong, S. He \\ School of Physics, Peking University, Beijing 100871, China \\ E-mail: liuchuan@pku.edu.cn, shendandan@gmail.com, \\ blake@pku.edu.cn, gzhmeng@pku.edu.cn, pkuFengXu@pku.edu.cn, \\ gongmingpkulgmail.com, fallenleave910@163.com
}

\section{Y. Chen, G. Li}

Institute of High Energy Physics, Academia Sinica, P. O. Box 918, Beijing 100039, China

E-mail: cheny@mail.ihep.ac.cn, gli@mail.ihep.ac.cn

\section{Y.B. Liu, X.F. Meng}

Department of Physics, Nankai University, Tianjin 300071, China

E-mail: liuyb@nankai.edu.cn, mengxf@mail.nankai.edu.cn

\section{J.P. Ma}

Institute of Theoretical Physics, Academia Sinica, Beijing 100080, China

E-mail: majpeitp.ac.cn

\section{J.B. Zhang}

Department of Physics, Zhejiang University, Hangzhou 310027, China

E-mail: jbzhangezimp.zju.edu.cn

We propose to study hadron-hadron scattering using lattice QCD in an asymmetric box which allows one to access more non-degenerate low-momentum modes for a given volume. The conventional Lüscher's formula applicable in a symmetric box is modified accordingly. To illustrate the feasibility of this approach, pion-pion elastic scattering phase shifts in the $I=2, J=0$ channel are calculated within quenched approximation using improved gauge and Wilson fermion actions on anisotropic lattices in an asymmetric box. After the chiral and continuum extrapolation, we find that our quenched results for the scattering phase shifts in this channel are consistent with the experimental data when the three-momentum of the pion is below $300 \mathrm{MeV}$. Agreement is also found when compared with previous theoretical results from lattice and other means. Moreover, with the usage of asymmetric volume, we are able to compute the scattering phases in the lowmomentum range (pion three momentum less than about $350 \mathrm{MeV}$ in the center of mass frame) for over a dozen values of the pion three-momenta, much more than using the conventional symmetric box with comparable volume.

The XXV International Symposium on Lattice Field Theory

July 30-4 August 2007

Regensburg, Germany

\footnotetext{
* Speaker.

${ }^{\dagger}$ For the CLQCD Collaboration
} 


\section{Introduction}

Hadron-hadron scattering experiments play a crucial role in understanding interaction among hadrons and QCD. Due to its non-perturbative feature, lattice QCD (LQCD) is the only systematic, non-perturbative method of QCD which in principle can be applied to calculate low-energy hadronhadron scattering from first principles. This type of lattice calculations is realized by a finite size method proposed by Lüscher [1]. Using this technique, the scattering length and the scattering phase shifts for pion-pion scattering in the $I=2, J=0$ channel have been studied [2, 3, 4, 5, 6, 7 , $8,9,10,11,12]$ in both quenched and unquenched lattice QCD.

However, non-degenerate low-momentum modes accessible for lattice simulation are usually limited due to computational cost. With cubic boxes, more non-degenerate low-momentum modes requires larger physical volumes. To circumvent this problem, we propose to use asymmetric boxes to study hadron-hadron scattering. The idea is tested in a quenched study of pion-pion scattering in the $I=2, J=0$ channel [24] using improved anisotropic lattices.

\section{Lüscher's formulae extended to an asymmetric box}

Consider a cubic box with size $L \times L \times L$ and periodic boundary condition. Three momentum of a single pion is quantized as: $\mathbf{k}=(2 \pi / L) \mathbf{n}$ with $\mathbf{n}=\left(n_{1}, n_{2}, n_{3}\right) \in \mathbb{Z}^{3}$, where $\mathbb{Z}$ represents the set of all integers. For the two-pion system taking the center of mass reference frame, we define $\overline{\mathbf{k}}^{2}$ of the pion pair in a box as:

$$
E_{\pi \pi}=2 \sqrt{m_{\pi}^{2}+\overline{\mathbf{k}}^{2}}
$$

where $E_{\pi \pi}$ is the exact energy of a two-pion system with the two pions having three momentum $\mathbf{k}$ and $-\mathbf{k}$ respectively in the center of mass frame. We further define $q^{2}$ via:

$$
\overline{\mathbf{k}}^{2}=\frac{4 \pi^{2}}{L^{2}} q^{2}
$$

With these definitions, Lüscher's formula for the $s$-wave scattering phase can be represented as:

$$
\cot \delta_{0}(\bar{k})=\frac{\mathscr{Z}_{00}\left(1, q^{2}\right)}{\pi^{3 / 2} q}
$$

where the so-called zeta function $\mathscr{Z}_{l m}$ is given by:

$$
\mathscr{Z}_{l m}\left(s, q^{2}\right)=\sum_{\mathbf{n}} \frac{\mathscr{Y}_{l m}(\mathbf{n})}{\left(\mathbf{n}^{2}-q^{2}\right)^{s}} .
$$

In this definition, $\mathscr{Y}_{l m}(\mathbf{r})=r^{l} Y_{l m}(\Omega \mathbf{r})$, with $Y_{l m}(\Omega \mathbf{r})$ being the usual spherical harmonics.

We need to extend the above formulae to an asymmetric box of physical size: $L \times \eta_{2} L \times \eta_{3} L$. The momentum in the box is quantized as: $\mathbf{k}=(2 \pi / L) \tilde{\mathbf{n}}$ with $\tilde{\mathbf{n}} \equiv\left(n_{1}, n_{2} / \eta_{2}, n_{3} / \eta_{3}\right)$ and $\mathbf{n}=$ $\left(n_{1}, n_{2}, n_{3}\right) \in \mathbb{Z}^{3}$. Quantities $\overline{\mathbf{k}}$ and $q^{2}$ are still defined according to Eq. (2.1) and Eq. (2.2). The formula for the scattering phase shifts is now modified to: [13, 14]

$$
\cot \delta_{0}(\bar{k})=m_{00}(q) \equiv \frac{\mathscr{Z}_{00}\left(1, q^{2} ; \eta_{2}, \eta_{3}\right)}{\pi^{3 / 2} \eta_{2} \eta_{3} q}
$$


with the modified zeta function $\mathscr{Z}_{l m}$ defined as:

$$
\mathscr{Z}_{l m}\left(s, q^{2} ; \eta_{2}, \eta_{3}\right)=\sum_{\mathbf{n}} \frac{\mathscr{Y}_{l m}(\tilde{\mathbf{n}})}{\left(\tilde{\mathbf{n}}^{2}-q^{2}\right)^{s}}
$$

In the large volume limit, the formula for scattering length is:

$$
E_{\pi \pi}-2 m_{\pi}=-\frac{4 \pi a_{0}}{\eta_{2} \eta_{3} m_{\pi} L^{3}}\left[1+c_{1}\left(\eta_{2}, \eta_{3}\right)\left(\frac{a_{0}}{L}\right)+c_{2}\left(\eta_{2}, \eta_{3}\right)\left(\frac{a_{0}}{L}\right)^{2}\right]+O\left(L^{-6}\right) .
$$

where the coefficients for the case $\eta_{2}=1$ and $\eta_{3}=2$, which is what we simulated in this work, are found to be: $c_{1}(1,2)=-1.805872, c_{2}(1,2)=1.664979$.

\section{Simulation details and results}

To test our idea of using the asymmetric box on hadron-hadron scattering, we perform a quenched study on the pion-pion scattering phase shift in the $I=2, J=0$ channel. In this section, we will briefly introduce our numerical results. More details can be found in Ref. [24].

\begin{tabular}{|c||c|c|c|}
\hline$\beta$ & Lattice & $a_{s}\left(\mathrm{GeV}^{-1}\right)$ & Confs. \\
\hline 2.080 & $8^{2} \times 16 \times 40$ & 1.5677 & 464 \\
\hline 2.215 & $9^{2} \times 18 \times 48$ & 1.3926 & 425 \\
\hline 2.492 & $12^{2} \times 24 \times 64$ & 1.0459 & 105 \\
\hline
\end{tabular}

The gauge action used in this study is the tadpole improved gluonic action on anisotropic lattices [16, 17] and the fermion action used in this calculation is the tadpole improved clover Wilson action on anisotropic lattice [18, 19, 20, 21, 22]. The anisotropy parameter $\xi=a_{s} / a_{t}$ is fixed to 5 for all our lattices. Some of the simulation parameters are provided in the table above.

To obtain the two-pion energy levels, we follow the standard procedure of correlation matrix measurements. For the single pion operators, we use:

$$
\pi^{+}(\mathbf{x}, t)=-\bar{d}(\mathbf{x}, t) r_{5} u(\mathbf{x}, t),
$$

The Fourier transformed fields are defined accordingly. The $s$-wave two-pion operators in the $I=2$ channel are defined as:

$$
\mathscr{O}_{n}(t)=\sum_{R} \pi_{R\left(\mathbf{k}_{n}\right)}^{+}(t) \pi_{R\left(-\mathbf{k}_{n}\right)}^{+}(t+1) .
$$

where the summation runs over all symmetry operations $R$ of the symmetry group for the asymmetric box. In the simulation, we measure the correlation matrix among different non-degenerate two-pion modes, using the two-pion operators defined in Eq. (3.2):

$$
\mathscr{C}_{m n}(t)=\left\langle\mathscr{O}_{m}^{\dagger}(t) \mathscr{O}_{n}\left(t_{s}\right)\right\rangle
$$

We then constructed a new correlation matrix: [23]

$$
\Omega\left(t, t_{0}\right)=\mathscr{C}\left(t_{0}\right)^{-\frac{1}{2}} \mathscr{C}(t) \mathscr{C}\left(t_{0}\right)^{-\frac{1}{2}}
$$

where $t_{0}$ is some suitable reference time. The eigenvalue $\lambda_{i}(t)$ of this new matrix $\Omega$ is:

$$
\lambda_{i}\left(t, t_{0}\right) \propto e^{-E_{i}\left(t-t_{0}\right)}
$$



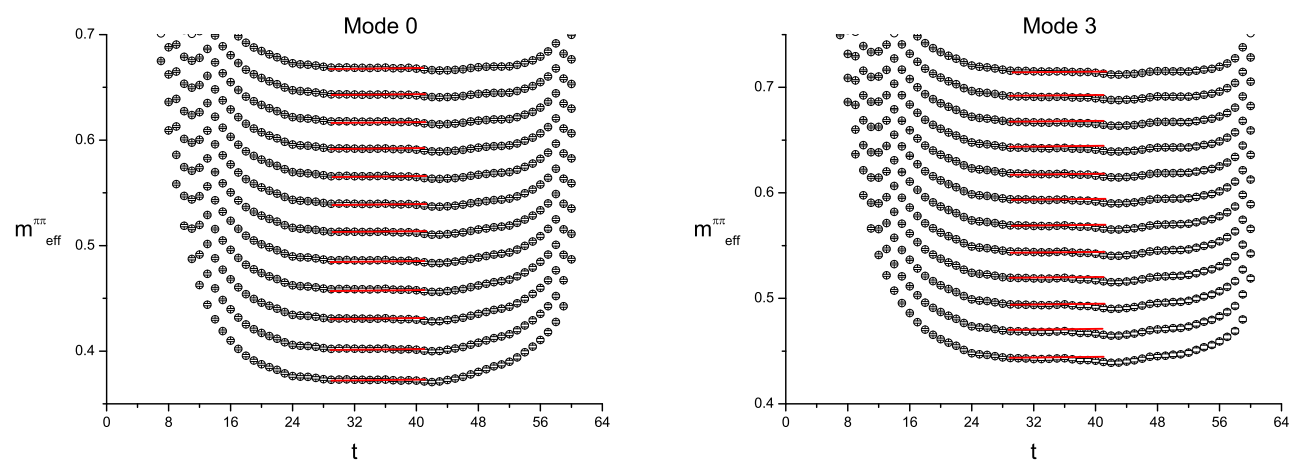

Figure 1: Effective mass plateaus of two-pion system at $\beta=2.492$ after the diagonalization procedure. The horizontal line segments in the figure represent the fitting ranges of the plateaus. Results for other values of $\beta$ are similar. Only two momentum modes out of 6 are shown.

from which the two-pion energy eigenvalues $E_{i}$ are obtained. In Fig. 1, we have shown the effective mass plateaus for the two-pion energies at $\beta=2.492$. The horizontal line segments designate the fitted values for the plateaus. Due to the usage of anisotropic lattices, good accuracy is achieved for two-pion energy levels.

In our quenched study, the pion mass values are beyond the applicable range of chiral perturbation theory. We therefore adopted polynomial parametrization for the data points for the scattering lengths and the phase shifts. For the scattering length, we parameterize our data for $a_{0} / m_{\pi}$ using either a linear function in $m_{\pi}^{2}$ :

$$
\frac{a_{0}}{m_{\pi}}=A_{0}+A_{1} m_{\pi}^{2}
$$

or, for the case of $\beta=2.492$ where a curvature is seen, a quadratic form in $m_{\pi}^{2}$. After the chiral
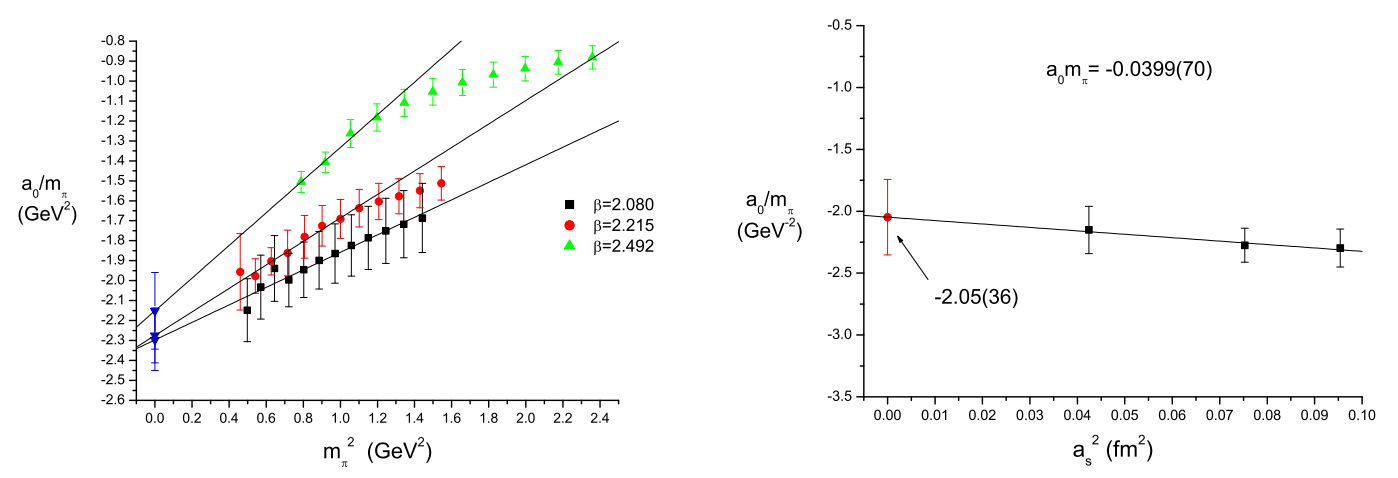

Figure 2: Left panel: chiral extrapolation of the quantity $\frac{a_{0}}{m_{\pi}}$. Right panel: the corresponding continuum limit extrapolation of the quantity $\frac{a_{0}}{m_{\pi}}$. The extrapolated result is indicated by a solid circle near $a_{s}=0$.

extrapolation, the results for the scattering length at different lattice spacing values are extrapo- 
lated towards the continuum with a linear function in $a_{s}^{2}$. The chiral extrapolation and the continuum extrapolation for the scattering length are shown in Fig. 2. As a result, we finally obtain: $a_{0} m_{\pi}=0.0359(59)$ which is compatible with results obtained in other lattice simulations, chiral perturbation theory and the experiment.

Similarly, we parameterize the phase shift $\delta$ itself by a polynomial in both $m_{\pi}^{2}$ and $\bar{k}^{2}$ as:

$$
\delta\left(m_{\pi}^{2}, \bar{k}^{2}\right)=D_{00}+D_{10} m_{\pi}^{2}+D_{20} m_{\pi}^{4}+D_{01} \bar{k}^{2}+D_{11} m_{\pi}^{2} \bar{k}^{2}+D_{02} \bar{k}^{4}
$$

The coefficient $D_{00}$ vanishes due to chiral symmetry which is checked numerically as well. A linear extrapolation in $a_{s}^{2}$ is then performed for various coefficients $D_{i j}$ appearing in the above equation. Some of the extrapolations are shown in Fig. 3 .
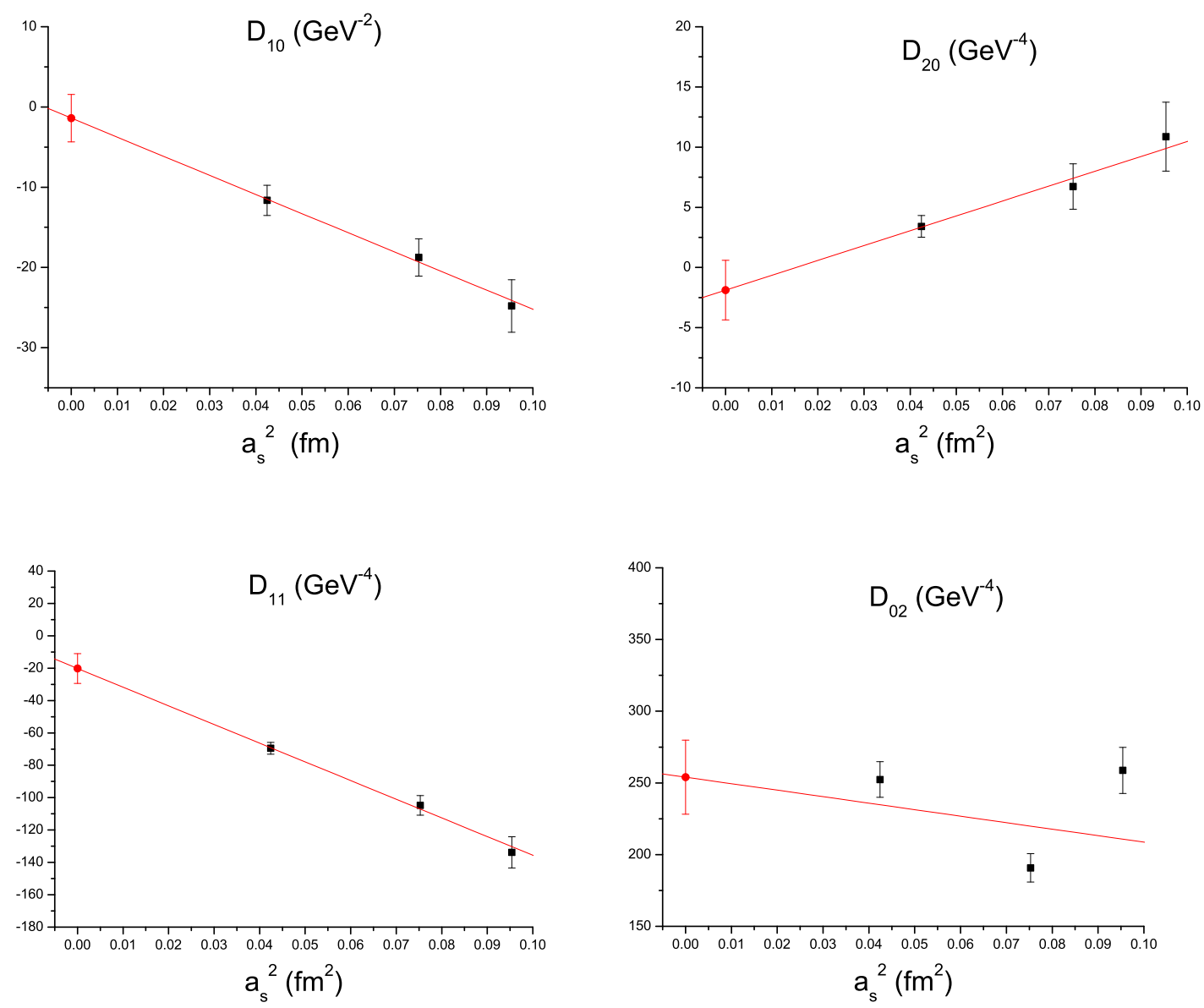

Figure 3: The continuum limit extrapolation of the parameter $D_{i j}$. The solid circles near $a_{s}=0$ represent the corresponding continuum limit values.

The results for the phase shift after the chiral and continuum extrapolation are depicted in Fig. 4, together with the experimental results from CERN-Munich group [15]. First, it is readily seen that, due to the usage of the asymmetric box, much more low-momentum modes are accessible with a relatively small volume, allowing better comparison with the experimental data in the lowmomentum range. Second, it is also found that our final results agree with the experimental results 


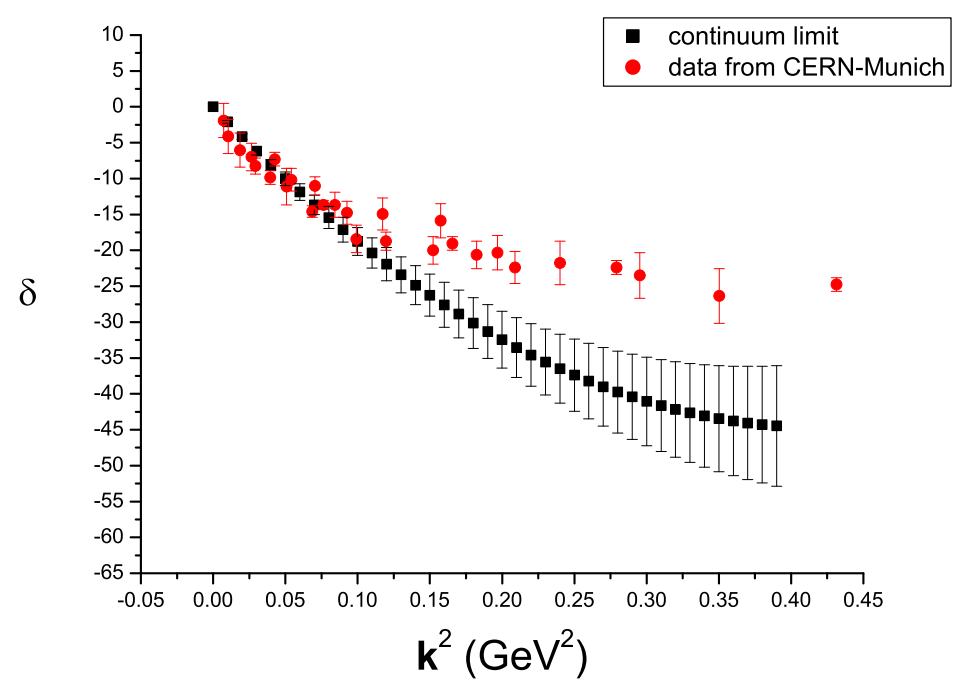

Figure 4: Comparison of our lattice results for the scattering phase shifts with the experimental data from CERN-Munich [15]. Results are consistent with each other for $\mathbf{k}^{2}$ below $0.1 \mathrm{GeV}^{2}$ which roughly corresponds to the center of mass energy of about $0.6 \mathrm{GeV}$.

within errors for $\overline{\mathbf{k}}^{2}$ below $0.1 \mathrm{GeV}^{2}$ which is about $\sqrt{s}=0.6 \mathrm{GeV}$. At higher energies, our results deviate from the experimental results. This deviation might be caused by the systematic uncertainties in our calculation, e.g. quenching and chiral extrapolations. Numerically speaking, it is largely due to poor determination of the coefficient $D_{02}$. However, we would like to point out that, it is clear from our quenched calculation that, the asymmetric volume technique advocated here would also be useful for unquenched studies once the unquenched configurations become available.

\section{Conclusions}

In this paper, we propose to study hadron-hadron scattering processes on lattices with asymmetric volume. This setup has the advantage that it provides much more non-degenerate lowmomentum modes with a relatively small volume, allowing more detailed comparison both with the experiments and with other theoretical results. The feasibility of this idea is illustrated in a quenched calculation of pion-pion scattering length and scattering phases in the $I=2, J=0$ channel using clover improved lattice actions on anisotropic lattices. Our quenched results indicate that the usage of asymmetric volumes indeed allow us to access much more low-momentum modes than in the case of cubic volume of similar size. For $\bar{k}^{2}$ in the range of $0.02 \mathrm{GeV}^{2}$ to $0.12 \mathrm{GeV}^{2}$, we have over a dozen of data points for the phase shift, much more than that in the cubic case with similar volume. It is also noted that, in the low-momentum region, after the chiral and continuum extrapolations, our results for the scattering length and the scattering phase shifts are in good agreement with the experimental data and are consistent with results obtained using other theoretical means. Although our calculation is now performed in the quenched approximation, similar calculations are 
also possible in the unquenched case once the gauge field configurations become available.

\section{Acknowledgments}

The authors would like to thank Prof. H. Q. Zheng and Prof. S. L. Zhu of Peking University for helpful discussions. The numerical calculations were performed on DeepComp 6800 supercomputer of the Supercomputing Center of Chinese Academy of Sciences, Dawning 4000A supercomputer of Shanghai Supercomputing Center, and NKstar2 Supercomputer of Nankai University.

\section{References}

[1] M. Lüscher. Nucl. Phys. B, 354:531, 1991.

[2] R. Gupta, A. Patel, and S. Sharpe. Phys. Rev. D, 48:388, 1993.

[3] M. Fukugita, Y. Kuramashi, H. Mino, M. Okawa, and A. Ukawa. Phys. Rev. D, 52:3003, 1995.

[4] S. Aoki et al. Nucl. Phys. (Proc. Suppl.) B, 83:241, 2000.

[5] S. Aoki et al. Phys. Rev. D, 66:077501, 2002.

[6] C. Liu, J. Zhang, Y. Chen, and J.P. Ma. Nucl. Phys. B, 624:360, 2002.

[7] P. Hasenfratz, K.J. Juge, and F. Niedermayer. JHEP, 0412:030, 2004.

[8] X. Du, G. Meng, C. Miao, and C. Liu. Int. J. Mod. Phys. A, 19:5609, 2004.

[9] S. Aoki et al. Phys. Rev. D, 71:094504, 2005.

[10] S. Aoki et al. Phys. Rev. D, 67:014502, 2003.

[11] T. Yamazaki et al. Phys. Rev. D, 70:074513, 2004.

[12] Silas R. Beane, Paulo F. Bedaque, Kostas Orginos, and Martin J. Savage. Phys. Rev. D, 73:054503, 2006.

[13] X. Li and C. Liu. Two particle states in an asymmetric box. Phys. Lett. B, 587:100, 2004.

[14] X. Feng, X. Li, and C. Liu. Two particle states in an asymmetric box and the elastic scattering phases. Phys. Rev. D, 70:014505, 2004.

[15] W. Hoogland et al. Nucl. Phys., B69:266-278, 1974.

[16] C. Morningstar and M. Peardon. Phys. Rev. D, 56:4043, 1997.

[17] C. Morningstar and M. Peardon. Phys. Rev. D, 60:034509, 1999.

[18] M. Alford, T. R. Klassen, and P. Lepage. Phys. Rev. D, 58:034503, 1998.

[19] T. R. Klassen. Nucl. Phys. (Proc. Suppl.) B, 73:918, 1999.

[20] Junhua Zhang and C. Liu. Mod. Phys. Lett. A, 16:1841, 2001.

[21] Shiquan Su, Liuming Liu, Xin Li, and Chuan Liu. Int. J. Mod. Phys. A, 21:1015, 2006.

[22] Wei Liu, Ying Chen, Ming Gong, Xin Li, GuoZhan Meng, and Chuan Liu. Mod. Phys. Lett. A, 21:2313, 2006.

[23] M. Lüscher and U. Wolff. How to calculate the elastic scattering matrix in two-dimensional quantum field theories by numerical simulation. Nucl. Phys. B, 339:222, 1990.

[24] X. Li et al. JHEP, 0707:053, 2007. 